\title{
Médias 2.0 et Églises chrétiennes au Burkina Faso
}

\author{
Évangélisation numérique \\ et contrôle du message
}

Louis Audet Gosselin

\begin{abstract}
[Résumé] La diffusion progressive d'Internet au Burkina Faso a entraîné récemment un foisonnement de l'utilisation des réseaux sociaux, principalement Facebook, par les Églises catholique et évangéliques. Cette utilisation est toutefois fortement conditionnée par un nombre restreint de gatekeepers qui exercent un contrôle souvent strict sur l'utilisation des réseaux sociaux. S'en dégage ainsi une diffusion fortement uniformisée par laquelle les organisations néo-pentecôtistes et charismatiques urbains tirent le plus grand profit en faisant circuler des contenus susceptibles de favoriser les conversions ou de souder virtuellement la communauté des adhérents. Les hiérarchies des Églises tendent plutôt, quant à elles, à utiliser ces médias comme relais de leur communication officielle sans investir dans le potentiel interactif des médias sociaux. Cette dynamique favorise également l'insertion des chrétiens burkinabè dans les réseaux chrétiens transnationaux, surtout pour certains pasteurs évangéliques inscrits dans les réseaux francophones internationaux.
\end{abstract}

Mots-clés : Burkina Faso, médias, catholicisme, pentecôtisme.

[Abstract] The gradual diffusion of Internet in Burkina Faso recently prompted an sharp increase in the use of social media, especially Facebook, by Catholic and Evangelical Churches. That use is however strictly controlled by a limited number of gatekeepers. The Burkinabè social media scene appears thus very uniform. Urban Evangelical and Charismatic Churches generally make the most active use of the potentialities of social media by diffusing content aimed at gaining new converts or strengthening the virtual faith community. Church hierarchies for their part mostly use social media as an offshoot of their regular communication strategy without much innovation. Social media also favours the connexion of Burkinabè Christians to global religious networks, especially a few Evangelical pastors who are well embedded in transnational Francophone networks.

Keywords: Burkina Faso, medias, Catholicism, Pentecostalism.

\footnotetext{
${ }^{1}$ Centre d'expertise et de formation sur les intégrismes et la radicalisation religieuse (CEFIR) (Canada)
} 


\section{Introduction}

Depuis la fin du $\mathrm{XX}^{\mathrm{e}}$ siècle, de nombreux écrits en sciences humaines et sociales ont traité des liens entre nouveaux mouvements religieux et médias, dans un contexte où les nouvelles technologies de l'information et de la communication ont transformé radicalement les modes de transmission des informations dans tous les domaines. L'étude de la religion dans les médias numériques est maintenant considérée comme un champ de recherche à part entière (Campbell, 2017), fondé sur plusieurs études surtout en langue anglaise, concernant les réseaux évangéliques (Gordon, 2005) et catholiques (Csordas, 2009). Sur le continent africain, les travaux de Birgit Meyer (2009) ont particulièrement marqué la lecture des rapports entre médias et religion. Rosalind Hackett avait auparavant souligné le rôle pionnier des Églises évangéliques nigérianes et ghanéennes dans l'utilisation des nouveaux médias (1998). Cependant, ce type d'études demeure rare dans le cadre burkinabè, où l'étude des médias est surtout centrée sur les formes traditionnelles telles que les journaux imprimés (Balima et Frère, 2003), et où l'étude de l'utilisation des médias par les organisations religieuses a surtout mis en évidence l'établissement de stations de radio et de télévision, ainsi qu'une implantation balbutiante sur les plateformes numériques (Madore, 2016 ; Savadogo et Gomez-Perez, 2011). Cette présence semble toutefois s'être renforcée depuis le début des années 2010, avec la diffusion des médias sociaux et l'accès accru des Burkinabè à Internet, autant dans le pays qu'au sein de la diaspora. Cet accès reste néanmoins limité, puisqu'on estimait au début 2017 qu'à peine plus de $10 \%$ des Burkinabè avaient accès à Internet ${ }^{2}$. Le phénomène étudié ici concerne donc une minorité en grande partie plus aisée que la moyenne et fortement urbanisée.

Cet article vise donc à étudier l'ancrage dans les médias numériques des Églises chrétiennes, (catholiques et évangéliques), ainsi que des groupes et individus s'en réclamant. Ces Églises évoluent en contexte minoritaire, dans un pays où $60 \%$ de la population est musulmane contre $19 \%$ de catholiques et près de $5 \%$ de chrétiens évangéliques selon le recensement de 2006 (INSD, 2009). Cependant, l'Église catholique a bénéficié d'une forte influence sur la formation des élites depuis l'époque coloniale et maintient une présence publique considérable, en plus d'afficher une croissance démographique constante (voir Kane, 2016 ; Brunel, 1996). De leur côté, les Églises évangéliques demeurent très minoritaires. Cependant, leur dynamisme est considérable et leur implantation en milieu urbain s'est grandement accrue depuis les années 1980, à l'instar de ce qui a été constaté à travers le monde. De plus, elles se distinguent par une action d'évangélisation très agressive qui assure une croissance rapide. Au Burkina Faso, la plus grande Église évangélique est celle des Assemblées de Dieu, établie

\footnotetext{
${ }^{2}$ Noufou Kindo, «Accès à Internet : le Burkina à la traîne », Burkina24.com, 31 janvier 2017, En ligne, consulté le 30 avril 2017, URL : https://burkina24.com/2017/01/31/taux-dacces-ainternet-le-burkina-trainent-toujours-les-pieds/.
} 
par des missionnaires américains en 1921, mais de nombreuses dénominations se sont créées depuis (Église de Pentecôte, Centre international d'évangélisation, églises locales indépendantes), souvent autour de la figure d'un pasteur charismatique et influent (Laurent, 2009; Samson, 2008 ; Fancello, 2006).

Cette étude s'appuie sur une collecte des productions médiatiques issues des Églises burkinabè depuis la fin des années 1990, sur tous les formats. Ces données ont été récoltées depuis plusieurs années de recherche auprès de ces églises (archives privées), dans les centres d'archives publiques ainsi que par un dépouillement systématique des contenus médiatiques en ligne (sites web, réseaux sociaux). Ces sources sont mises en perspective avec les enquêtes ethnographiques réalisées au sein de diverses églises catholiques et évangéliques burkinabè depuis 2010. Notre recherche s'ancre dans la théorie médiatique du " gatekeeping », qui permettra d'aborder le rôle d'acteurs clés du monde des médias qui agissent en véritables gardiens de l'accès à la prise de parole et des enjeux mis de l'avant dans les médias (Lewin, 1947; Shoemaker, 1991). Loin de remettre en cause cette dynamique, l'arrivée des médias sociaux au $\mathrm{XXI}^{\mathrm{e}}$ siècle a plutôt permis l'émergence de nouveaux gatekeepers (Barzilai-Nahon, 2008). Ainsi, plutôt que de devenir l'utopie d'une circulation libre et décentralisée des contenus médiatiques, les médias « 2.0 » auraient simplement déplacé les lignes de partage entre production, circulation et réception des médias, en plus de brouiller les pistes quant aux origines souvent très concentrées des contenus.

Cette perspective semble s'appliquer de façon très claire en ce qui concerne les Églises chrétiennes, autant catholique qu'évangéliques, au Burkina Faso. En effet, la diffusion des messages semble remarquablement uniforme et les voix discordantes ou alternatives demeurent marginales. D'une part, les institutions ecclésiales produisent et diffusent, sur de multiples plateformes, un contenu autorisé pour lequel les médias traditionnels autant que sociaux font office de caisse de résonnance. D’autre part, les voix indépendantes existent et se font entendre à divers degrés, mais leur succès semble dépendre fortement de leur adéquation avec les objectifs des gatekeepers autorisés.

Dans un premier temps, nous présenterons les stratégies médiatiques de l’Église catholique, en soulignant la centralisation de la communication autour d'organisations proches de l'épiscopat, ainsi que l'utilisation des médias sociaux comme prolongement de cet effort centralisé, tout en laissant la place à quelques initiatives autonomes. Dans un second temps, nous montrerons que les Églises évangéliques, plus décentralisées et divisées en de multiples tendances, font également preuve d'une surprenante centralisation de la communication autour de quelques pôles de production, tout en maintenant une apparence de spontanéité. De plus, les échanges sur les médias sociaux évangéliques burkinabè sont généralement consensuels et visent à renforcer une cohésion interne. 


\section{L'Église catholique : centralisation et essor charismatique}

L'Église catholique burkinabè dispose d'une stratégie de communication centralisée autour de la hiérarchie, principalement celle de Ouagadougou, qui se transpose dans la sphère numérique. En parallèle de cette stratégie centralisée, certains groupes catholiques indépendants (Jeunesse étudiante catholique, renouveau charismatique) développent leur propre présence sur les médias sociaux, présence qui permet l'expression de points de vue quelque peu marginaux dans l'Église, sans remettre fondamentalement en question le contrôle de la hiérarchie sur les orientations générales de la communication catholique 2.0.

\subsection{Stratégies ecclésiales et centralisation des communications}

Depuis le début du $\mathrm{XXI}^{\mathrm{e}}$ siècle, le clergé catholique burkinabè a entrepris d'investir l'espace numérique et ainsi maintenir sa visibilité publique. La création du site web de l'Église du Burkina Faso en 2005 constitue une première pour une grande organisation religieuse dans ce pays. Ce site, revampé depuis ${ }^{3}$, centralise les actualités de l'Église nationale, les principales communications des prélats nationaux et du Pape, en plus de rassembler certains documents de l’Église. Il publie en majorité des textes issus du service de communication de l'Église, qui souvent paraissent également sur l'un des principaux organes de presse numérique burkinabè, lefaso.net. Ce site constitue un outil de diffusion de masse des activités religieuses : prises de positions par rapport aux événements sociopolitiques, informations à propos des fêtes et célébrations religieuses, principales homélies, de même qu'échos des nouvelles vaticanes. À travers la large diffusion des articles de lefaso.net sur les médias sociaux grâce à ses 230000 abonnés Facebook et 44.000 abonnés Twitter, l'Église peut donc rejoindre une large part des internautes du Burkina Faso et de la diaspora.

Cette stratégie semble tirer son origine d'un cercle restreint d'acteurs proches de la hiérarchie, en particulier du Cardinal Philippe Ouédraogo. En effet, le Service pastoral pour la formation et l'accompagnement des responsables (SEPAFAR) a été mis en place en 2011 par le Cardinal, lors de l'érection de l'aumônerie des étudiants et élèves en paroisse universitaire. Le SEPAFAR vise à organiser l'apostolat des élites politiques du pays. Il a notamment joué un rôle important dans la critique du régime de Blaise Compaoré et certains de ses membres occupent des postes très influents dans la donne politique post-insurrection. C'est le cas d'Augustin Loada, ministre de la Fonction publique en 2014-2015, d'Armand Béouindé, maire de Ouagadougou depuis juin

\footnotetext{
${ }^{3}$ Consulté le 26 avril 2017, URL : www.egliseduburkina.org.
} 
2016 et de Clément P. Sawadogo, secrétaire général du Mouvement du peuple pour le progrès (MPP), au pouvoir depuis 2015, et ministre du travail et de la fonction publique depuis $2016^{4}$. Ce groupe fait un usage significatif des médias sociaux pour ses communications externes et internes, avec la mise sur pied d'un groupe Yahoo pour les membres dès sa création, suivi d'une page Facebook en $2015^{5}$, régulièrement alimentée et suivie par près de 500 personnes ${ }^{6}$.

Associé au SEPAFAR, on retrouve la communauté des Augustins de l'assomption, communément appelés Assomptionnistes, implantés au Burkina Faso depuis 2011 en réponse à la volonté de l'épiscopat de moderniser les communications catholiques. L'une de leurs principales attributions est l'implantation de médias catholiques, la congrégation étant propriétaire de la maison d'édition Bayard. C’est sous son impulsion que l'archidiocèse de Ouagadougou a lancé en 2016 le journal mensuel papier Duc in altum ${ }^{7}$. Le père Jean-Paul Sagadou, supérieur des Assomptionnistes du Burkina Faso, s'implique au SEPAFAR, dont il anime certaines des messes lors des absences de l'aumônier, en plus d'officier à titre de responsable des communications ${ }^{8}$.

Ainsi, l'arrivée de Mgr Philippe Ouédraogo comme archevêque de Ouagadougou en 2009 a accéléré la refonte de l'action médiatique catholique, en particulier son implantation plus active dans la sphère numérique et sur les réseaux sociaux afin de mobiliser les fidèles, en particulier ceux appartenant aux élites économiques et intellectuelles. Cette initiative se double d'entreprises autonomes de la part d'organisations catholiques de jeunes, principalement étudiants et élèves, qui utilisent les médias sociaux pour véhiculer leurs messages et mobiliser leurs réseaux.

\subsection{Acteurs catholiques indépendants : entre conformisme et dynamisme}

Aux côtés des publications émanant de la hiérarchie elle-même et de son service de communication, on constate l'existence d'une présence autonome de certains groupes catholiques sur les médias sociaux, principalement scolaires et estudiantins de la capitale. La Jeunesse estudiantine catholique (JEC) constitue une institution phare de-

\footnotetext{
${ }^{4}$ SEPAFAR (5 décembre 2016), « Bureau de coordination 2017 » [statut Facebook], consulté le 10 décembre 2017, URL : www.facebook.com/Sepafar-1594166820820989/?fref=ts.

${ }^{5}$ Noufou Kindo, "Commission de la réconciliation nationale : Une "force de proposition" se hisse», Burkina24.com. En ligne, consulté le 26 avril 2017, URL: https://burkina24.com/2015/02/05/commission-de-la-reconciliation-nationale-une-forcede-proposition-se-hisse/.

${ }^{6}$ Consulté le 26 avril 2017, URL : www.facebook.com/Sepafar-1594166820820989/?fref=.

${ }^{7}$ Nicole Ouédraogo, «Médias catholiques : Duc in Altum désormais journal d'informations générales », leFaso.net, 13 avril 2016.

${ }^{8}$ Entretien avec le père Jean-Paul Sagadou, supérieur des Assomptionnistes du Burkina, le 20 avril 2014, Ouagadougou.
} 
puis sa création dans les années 1950. Organe représentatif des élèves catholiques des lycées et collèges, l'association est également présente à l'Université de Ouagadougou, où elle a longtemps constitué la principale voix catholique. Organisation d'intellectuels, la JEC a mis sur pied des journaux dès sa création afin de diffuser des positions parfois en décalage avec celles de la hiérarchie. C'est donc sans surprise que l'activisme jéciste s'est transposé de façon autonome sur Internet. La JEC a donc lancé son site web en 2010 :

II n'était plus possible de rester longtemps sans ce moyen de communication pour notre mouvement. Un site, c'est un lieu ouvert où nous pouvons mieux faire connaître l'identité de notre mouvement et rentrer en dialogue avec d'autres personnes pour que le Règne de Dieu arrive dans notre vie. C'est un lieu où nous pourrons mettre en œuvre notre méthode de travail à travers la trilogie VOIR-JUGER-AGIR. Nous prenons donc ce qu'il y a de mieux dans les nouvelles technologies de notre monde dit post-moderne pour, à notre manière, moderniser notre mouvement. Je souhaite que ce site soit un portail ouvert par lequel et à travers lequel notre mouvement pourra aller « plus loin», toujours « plus loin ».

Le site, rarement mis à jour, est devenu inopérant entre 2012 et 2013 avant d'être remplacé à la rentrée 2013 par une page Facebook officielle qui est toujours active ${ }^{10}$.

Aux côtés de cette présence institutionnelle centrale, d'autres pages web ainsi que des pages et des groupes Facebook sont régulièrement lancés par des sections locales de la JEC, ou par d'autres organisations estudiantines (Mouvement eucharistique des jeunes, communautés chrétiennes de base des sections universitaires, chorales). De manière symptomatique pour des organisations avec un rapide roulement de militants, ces plateformes sont alimentées de façon irrégulière et tombent fréquemment en dormance, pour être réanimées par de nouvelles équipes. Par exemple, la page Facebook de la JEC du Burkina Faso n’a rien publié en 2015 ni en 2016, pour être subitement ravivée en 2017 en vue de la préparation de la semaine nationale de l'association.

Alors que les productions émanant de la hiérarchie, diffusées dans les médias généralistes, participent aux débats sociaux et politiques burkinabè, les publications web de la JEC et des organisations estudiantines visent essentiellement à diffuser les activités internes des associations afin de maintenir la mobilisation des élèves et étudiants catholiques. Cette orientation tranche avec l'attitude historique des publications de la JEC depuis les années 1950, dans lesquelles les militants n’hésitaient pas à

\footnotetext{
${ }^{9}$ " Mots de bienvenue du Secrétaire national de la JEC ». Consulté le 6 octobre 2010, URL : jec-burkina.org.

${ }^{10}$ Consulté le 25 avril 2017, URL : www.facebook.com/jecburkina/?fref=ts.
} 
aborder les questions sociopolitiques ${ }^{11}$. Ce changement semble indiquer un déclin de dynamisme de cette organisation, qui n'a plus le monopole de la présence catholique en milieu scolaire et estudiantin.

En effet, une organisation catholique estudiantine se démarque par son dynamisme dans les médias numériques : le Buisson Ardent, section universitaire du renouveau charismatique. Cette tendance du catholicisme, née sur les campus américains dans les années 1960 et influencée par la mouvance pentecôtiste (Csordas, 1994), connaît une popularité certaine au Burkina Faso, en particulier autour de l'abbé Blaise Bicaba. Ses séances d'évangélisation, qui s'accompagnent de guérisons miraculeuses et de conversions, drainent des foules considérables ${ }^{12}$. Cet engouement se répercute à plus petite échelle dans les activités des groupes charismatiques locaux. Par exemple, des groupes charismatiques paroissiaux attirent un grand nombre de fidèles, dont plusieurs recherchent la guérison de leurs problèmes lors de séances d'imposition des mains par les responsables ${ }^{13}$. Sur le campus universitaires, le Buisson Ardent attire quelques centaines de fidèles chaque semaine lors de séances de prières et d'adoration particulièrement intenses sur le campus de l'Université de Ouagadougou $^{14}$. Le groupe publie un feuillet papier appelé Le Flambeau et dispose d'un site web depuis $2011^{15}$. Par ailleurs, la page Facebook du Buisson Ardent est suivie par plus de 4000 personnes ${ }^{16}$, nettement plus que toute autre organisation catholique burkinabè. Enfin, le groupe dispose d'un compte Twitter qui relaie ses activités ${ }^{17}$.

L'utilisation des médias sociaux et numériques par ce groupe diffère de la JEC et des organisations liées à la hiérarchie. En effet, si le Buisson Ardent publicise ses activités pour les membres, il y diffuse surtout des extraits photo, vidéo et retranscriptions de prêches et d'activités d'évangélisation. Par exemple, la campagne d'évangélisation d'avril 2017 a fait l'objet de nombreuses communications sur toutes ces plateformes, avec une insistance particulière sur les guérisons miraculeuses survenues le 30 avril lors de la grande séance au Stade du 4 août, le plus grand du pays ${ }^{18}$. Ces communica-

\footnotetext{
${ }^{11}$ Fonds $\mathrm{JECl}$, Bibliothèque de documentation internationale contemporaine, FDelta 1980377 à 381 ; archives privées de la JEC universitaire du Burkina Faso, consultées le $1^{\text {er }}$ juillet 2010, aumônerie des élèves et étudiants de Ouagadougou.

${ }^{12}$ Observation d'une séance de prière de l'abbé Bicaba, église de Tampouy, Ouagadougou, le 19 février 2011.

${ }^{13}$ Observation d'une séance de renouveau charismatique paroissial, paroisse de KologhNaba, Ouagadougou, le 10 janvier 2011.

${ }^{14}$ Observations de séances du Buisson Ardent, Université de Ouagadougou, le 28 mai 2010 ; le 6 février 2015.

${ }^{15}$ Consulté le 26 avril 2017, URL : www.rccuo.com.

${ }^{16}$ Consulté le $1^{\text {er }}$ mai 2017, URL : www.facebook.com/rccuobuissonardent.

${ }^{17}$ Consulté le ${ }^{\text {er }}$ mai 2017. URL : https://twitter.com/buissonardent.

${ }^{18}$ Buisson Ardent Université de Ouagadougou ( ${ }^{\text {er }}$ mai 2017), « Grande campagne

d'évangélisation du Buisson Ardent au stade du 4 Août \# Jesus se retournant la vit [sic] et
} 
tions ont été le fait d'une équipe média nombreuse, qui a elle-même été présentée sur les plateformes du groupe ${ }^{19}$. En mettant en scène ce groupe de jeunes connectés, faisant un usage quasiment -professionnel de matériel technologique de pointe, le Buisson Ardent se pose ainsi comme une organisation en phase avec les technologies de pointe et en croissance.

L'Église catholique fait donc preuve d'une présence active sur les médias numériques. D'une part, cette présence est centralisée autour du clergé ouagalais et de groupes d'apostolat des laïcs, avec l'objectif d'influencer les débats sociopolitiques et de maintenir la présence de l'Église dans l'espace public. D'autre part, des organisations catholiques autonomes comme la JEC et le renouveau charismatique disposent de leurs propres organes de médias sociaux, utilisés pour maintenir une cohésion interne et attirer de nouveaux membres. La situation dans les Églises évangéliques est en partie similaire, avec une centralisation des producteurs, doublée d'une forte insertion dans les réseaux internationaux.

\section{2. Églises évangéliques : foisonnement apparent, messages canalisés et communication globalisée}

Aux côtés de l'Église catholique, historiquement très puissante, les Églises de la mouvance évangélique ont gagné en influence et visibilité depuis les années 1980 (Laurent, 2009; Fancello, 2006). Bien qu'en principe décentralisées, ces Églises font toutefois preuve d'une utilisation conservatrice des médias sociaux, avec des gatekeepers qui orientent l'ensemble de la communication. De plus, les réseaux sociaux évangéliques burkinabè s'appuient fortement sur des réseaux francophones transnationaux, accentuant l'uniformité des messages évangéliques sur la toile.

\subsection{Des Églises en vase clos}

Bien que treize Églises soient membres de la Fédération des Églises et missions évangéliques du Burkina, sans compter une kyrielle de petites Églises indépendantes, la mouvance évangélique fait montre d'une uniformité qui peut surprendre de prime abord dans ses communications numériques. En effet, malgré la multiplicité des acteurs, les messages véhiculés sont plutôt similaires. Une poignée d'organisations évangéliques sont dans les faits actives sur Internet. En premier lieu, le champ numé-

lui dit: "Ait confiance, ma fille, ta foi t'a sauvée" Mt9.22 » [statut Facebook], Consulté le 10 décembre 2017, URL : www.facebook.com/rccuobuissonardent/?fref=ts.

${ }^{19}$ Buisson Ardent Ouaga [@buissonardent], «L'Équipe du site web du Buisson Ardent, ces frères et sœurs qui ont œuvrés [sic] à relayer les temps forts de la Veillée Nationale. » [Tweet], consulté le 10 décembre 2017, URL :

https://twitter.com/buissonardent/status/858925615482900480. 
rique est dominé par les organes du Centre International d’Évangélisation (CIE) du pasteur Mamadou Karambiri. Produites dans un immeuble appelé Rhema Media Center, les communications du CIE incluent des publications de journaux papier (Impact Magazine), de livres du pasteur Karambiri, une station de télévision retransmise en ligne (ImpactTV $)^{20}$, ainsi qu'un site web ${ }^{21}$. Ces outils diffusent en priorité les prêches du pasteur, qui remportent un succès sans égal au Burkina Faso et ailleurs dans le monde francophone (Samson, 2008). De façon quelque peu surprenante, le CIE s'est implanté plus tardivement sur les médias sociaux, avec l'ouverture de la page Facebook «CIE-MIA Officiel » en janvier 2017, comptant quelques 700 abonnés, qui rediffuse les cultes du pasteur Karambiri et annonce les activités de l’Église. Le site web diffuse également pour l'essentiel les prêches du pasteur et de sa femme Hortense, également pasteure du CIE, en plus de fournir des informations sur l'Église et ses implantations. On peut y voir un signe d'une volonté de contrôle des messages par les gatekeepers du CIE, qui préfèrent éviter l'interaction et les possibles contradictions. Ce type d'utilisation des médias ressemble par ailleurs à celui opéré par la hiérarchie catholique, en s'appuyant en priorité sur des infrastructures «lourdes » (studios, imprimeries) et en centralisant la production et la diffusion.

L’Église des Assemblées de Dieu (AD), de loin la plus grande de la mouvance évangélique au Burkina Faso, contraste avec le CIE par une action médiatique plus limitée. Elle imprime le magazine bilingue français-mooré Flamme en couleurs et sur papier glacé, ainsi que d'autres livrets et publications ponctuelles dans les deux langues. Ces publications sont vendues lors des cultes et activités des églises $\mathrm{AD}$, ainsi que dans les librairies affiliées à cette dénomination (église centrale de Ouagadougou et mission américaine dans le quartier Gounghin). Sur Internet, le site web national des AD n'est plus fonctionnel et l'essentiel de la présence se trouve sur Facebook. C'est surtout sur ce réseau social que les $\mathrm{AD}$ sont actives, avec une page pour l'Église nationale et des pages pour les principales églises locales de la capitale (église centrale, église Zone pilote I, église de Gounghin, section jeunesse de l'église d'Antenne-Ville). Ces pages comptent plus de 1000 abonnés chacune, ce qui doit être nuancé par le fait que de nombreux fidèles suivent plusieurs pages évangéliques. Le temple Eben-Ezer, plus grande église $\mathrm{AD}$ de la région de Bobo-Dioulasso, possède également une page Facebook et un site web de facture professionnelle. Les publications sur les pages des AD sont souvent irrégulières. Elles concernent essentiellement les activités des églises, avec un accent mis sur les activités particulières : fêtes, visites de pasteurs étrangers, journées dédiées des sections (femmes, jeunes, chorales), en plus de relayer certains prêches des pasteurs principaux.

\footnotetext{
${ }^{20}$ Consulté le 30 avril 2017, URL : www.impacttele.tv.

${ }^{21}$ Consulté le 30 avril 2017, URL : www.cie-mia.org.
} 
La page Facebook de l'Église de l'Alliance Chrétienne de Ouagadougou compte plus de 1000 abonnés. Cependant, outre les annonces du décès d'un jeune membre et d'une campagne d'évangélisation en mars 2017, elle ne contient pour l'essentiel que des liens vers les "versets du jour " générés automatiquement sur la page web de l'église. La section ouagalaise de cette Église basée à Bobo-Dioulasso constitue sa seule présence en ligne, ce qui souligne la concentration quasiment complète de la diffusion web dans la capitale. Quelques autres dénominations de la capitale (Église de Pentecôte de Somgandé, Église de la mission apostolique de Pissy, Église biblique de la Vie profonde, Église protestante évangélique) possèdent également des présences sur Facebook qui relaient de façon irrégulière les prêches des pasteurs et les activités internes des Églises à quelques centaines d'internautes.

Les élèves et étudiants évangéliques, regroupés dans l'organisation interdénominationnelle de l'Union des Groupes Bibliques du Burkina (UGBB), disposent également de leurs médias numériques. Après de longs délais, le projet de lancement d'un site web de l'UGBB a finalement été concrétisé en $2016^{22}$. De plus, la structure est présente sur Facebook où elle fait la promotion de ses activités, relaie des photos et vidéos de celles-ci, en plus de partager des nouvelles des membres importants (mariages, naissances). De façon générale, bien que la page de l'UGBB ne compte que 1150 abonnés en date du 30 avril 2017, les réactions y sont plus nombreuses et élaborées que sur les pages des Églises, ce qui peut témoigner soit d'une plus grande proximité entre les militants, soit d'un niveau d'activité plus grand sur la page.

En effet, de façon quasiment systématique, les réactions aux publications sur les pages évangéliques et catholiques consistent surtout en quelques formules religieuses. En plusieurs années de consultation des médias chrétiens, nous n'avons constaté aucun commentaire critique sur ces pages, chaque publication suscitant quelques « Amen » ou « Union de prières » et partages de la part de fidèles. Il est vraisemblable que ces pages soient surveillées par des modérateurs qui bannissent tout commentaire déviant. Cependant, ce conformisme s'explique également par la présence exclusive de fidèles dévoués sur ces réseaux, dans la mesure où il reproduit des comportements et attitudes maintes fois observés par l'auteur dans les activités des églises. L'espace numérique constitue donc un prolongement de la vie religieuse « réelle » afin de diffuser les prêches à des audiences plus larges. Ces réactions montrent qu'en dépit de l'apparente interactivité des médias sociaux, le contenu reste fortement unidirectionnel et contrôlé par les gatekeepers. Une telle uniformité n'est d'ailleurs pas propre au milieu évangélique burkinabè et a été constatée à travers le monde, où les voix discordantes sont presque inaudibles dans les médias évangéliques (Oosterbaan, 2017).

\footnotetext{
${ }^{22}$ Consulté le 30 avril 2017, URL : www.ugbb.org.
} 
Bien qu'inégal, l’investissement des médias numériques par les Églises évangéliques semble être un objectif important pour les églises urbaines comptant une proportion importante de fidèles scolarisés et connectés à Internet. Cette insistance sur la diffusion la plus large possible de la parole de Dieu pousse naturellement les Églises à s'internationaliser. Ainsi, la communication numérique évangélique fait une place importante aux connexions internationales, qui renforcent l'attrait et le prestige des Églises.

\subsection{Connexions internationales}

Les médias évangéliques mettent particulièrement de l'avant les connexions internationales des Églises et pasteurs burkinabè. Les campagnes d'évangélisation mettant en vedette des pasteurs étrangers figurent donc en bonne place dans les publications en ligne des Églises évangéliques ${ }^{23}$. Ces publications servent alors dans un premier temps de publicité pour attirer les fidèles à la prestation en personne et ainsi assurer une large audience. Dans un second temps, des photographies et surtout des vidéos sont partagés via toutes les plateformes disponibles. Le but de cette diffusion est double. D'une part, on cherche à relayer la puissance charismatique et persuasive de ces prédicateurs à un plus grand nombre de fidèles, en comptant sur les partages des adhérents dans leurs propres réseaux, qui comptent souvent à la fois des chrétiens évangéliques et des membres d'autres confessions, anciens camarades de classe, parents et collègues de travail. D’autre part, les images et vidéos mettent en scène la foule présente lors de l'événement. Les participants sont en effet actifs par leurs réactions (chants, transes, guérisons). De plus, l'ampleur de la foule constitue en ellemême un témoignage de la puissance et de l'influence des orateurs et de l'Église qui les accueille.

Les connexions internationales des Églises sont également affichées dans la reprise de contenus de plateformes internationales francophones, qui permettent de mettre en lien les membres de l'église locale avec les pasteurs les plus influents. Ces plateformes incluent en premier lieu le réseau TopChrétien. Ce site web ${ }^{24}$, fondé dès 1999 par Éric Célérier, un protestant évangélique français, se veut d'abord un agrégateur de contenu qui répertorie diverses ressources évangéliques en français. Son succès est impressionnant, puisqu'il recevrait plus d'un million de visites chaque mois (Kirschleger, 2015). Le réseau TopChrétien compte par ailleurs 647000 abonnés Face-

\footnotetext{
${ }^{23}$ Par exemple : Temple Eben Ezer Sarfalo (17 novembre 2016), « Grande campagne d'évangélisation Bobo-Dioulasso 2016 avec l'évangéliste Daniel Kolenda » [statut Facebook], consulté le 10 décembre 2017, URL :

www.facebook.com/profile.php?id=100010791547958\&pnref=story.

${ }^{24}$ Consulté le 30 avril 2017, URL : www.topchrétien.com.
} 
book et 19000 abonnés Twitter25. Il offre des applications mobiles, des versets quotidiens, une section pour enfants et des ressources pour orienter quiconque voudrait se convertir dans n'importe quel endroit de la francophonie et au-delà. Au Burkina Faso, c'est surtout le pasteur Mamadou Karambiri qui est en lien avec ce réseau. Il fait en effet partie du conseil de l'organisation et le CIE reçoit régulièrement des pasteurs populaires sur TopChrétien, en particulier le pasteur français Yvan Castanou, leader de l'Église Impact Centre Chrétien. Les fidèles évangéliques de base sont également très actifs dans la circulation de ces messages, qui deviennent ainsi inévitables pour tout fidèle présent sur les réseaux sociaux.

Certains pasteurs burkinabè enfin mettent en évidence leur propre rayonnement international. Plusieurs travaux ont souligné l'importance de l'imaginaire global dans les Églises évangéliques, où des dénominations modestes prennent souvent des noms pompeux évoquant une présence globale (Corten, 2006; Martin, 2001). Par ailleurs, la moindre implantation à l'étranger est mise en avant comme un rayonnement global par les Églises. Par exemple, le CIE clame que «par l'action de son département missionnaire, il a ouvert des églises au Niger, au Bénin, en Côte d'Ivoire, en Guinée Conakry, au Ghana, en France, en Italie, aux États-Unis d'Amérique et en République Démocratique du Congo ${ }^{26}$ ». Certaines de ces missions comptent une poignée de membres, essentiellement issus de la diaspora burkinabè. Toutefois, le rayonnement personnel du pasteur Karambiri est réel. Ses prêches sont depuis longtemps été diffusés sur le site TopChrétien, ainsi que sur Enseignemoi.com, un portail similaire fondé en France en 2004 et hébergé au Canada depuis 2010. Ses prêches sont également relayés sur les sites web et médias sociaux de certains des pasteurs les plus en vue en Europe francophone, qui lui assurent une visibilité transnationale considérable.

L'exemple du pasteur Karambiri est toutefois quasiment unique dans le champ religieux burkinabè et met en lumière une distinction au sein du milieu "protestant » burkinabè. Cette distinction sépare le courant pentecôtiste historique, incarné principalement par l'Église des Assemblées de Dieu, de la mouvance «néopentecôtiste» à laquelle se rattache Mamadou Karambiri. Alors que le premier courant s'appuie sur un cadre institutionnel fort, limite l'émergence de figures charismatiques individuelles et insiste sur le respect de la rigueur doctrinale, le second se fonde, depuis ses débuts aux États-Unis dans les années 1960, sur des personnalités fortes qui transcendent les cadres institutionnels. De plus, le néopentecôtisme est fortement associé à l'utilisation intensive des moyens médiatiques de pointe ainsi qu'à la création de réseaux transnationaux basés sur des liens personnels entre pasteurs charismatiques plutôt que sur la croissance internationale des institutions comme dans le cas des Assemblées de Dieu (Mossière, 2014 ; Fancello, 2006).

\footnotetext{
${ }^{25}$ Portails consultés le 29 avril 2017.

${ }^{26}$ Consulté le 30 avril 2017, URL : www.cie-mia.org/qui-sommes-nous.
} 
Le rayonnement international des pasteurs burkinabè, ainsi que la connexion des Églises nationales aux réseaux internationaux, participe donc directement d'une théologie évangélique et missionnaire, qui ordonne d'aller propager la Bonne Nouvelle dans «toutes les nations» (Fancello et Mary, 2010). Ces connexions internationales sont surtout visibles au sein de la mouvance "néopentecôtiste " incarnée en premier lieu par le pasteur Mamadou Karambiri. Il s'ajoute et prolonge naturellement une implantation des églises locales urbaines burkinabè dans l'espace numérique en rehaussant le prestige de ces dernières, en faisant porter leur message à l'extérieur des frontières et en mettant en avant leur modernité.

\section{Conclusion}

On constate donc le rôle central joué par les gatekeepers chrétiens dans la circulation du message évangélique sur le web. Ces derniers ont l'initiative sur le type de contenu diffusé, la forme de celui-ci, ainsi que sur l'orientation générale de la réception et des interactions des fidèles. En diffusant d'abord auprès de membres convaincus, en choisissant des contenus avantageux et éventuellement en modérant les réactions, ces gatekeepers créent les conditions d'un espace médiatique unifié malgré la multiplicité des acteurs. Cet espace sert d'une part à renforcer la cohésion des groupes déjà établis et d'autre part à susciter de nouvelles conversions par la diffusion de contenu convaincant auprès de publics ciblés (amis et collègues de fidèles, étudiant-e-s). La transition progressive des sites web classiques vers les médias sociaux, principalement Facebook, ne semble pas radicalement modifier la donne, les possibilités interactives de ces médias servant surtout de caisse de résonnance au contenu développé et diffusé par les gatekeepers.

Notre étude relève toutefois des utilisations diversifiées des médias numériques par les Églises et les organisations chrétiennes burkinabè, différences qui transcendent en partie le clivage catholique/évangélique. Dans un premier temps, l'Église catholique dispose d'une coordination médiatique centralisée autour de la hiérarchie et de groupes spécialisés, implantés dans les milieux d'élite de la capitale. Ce fonctionnement, qui s'apparente à celui du Centre International d'Évangélisation, utilise les médias numériques comme prolongement d'une stratégie de communication professionnalisée et basée en premier lieu sur des médias conventionnels (radios, télévisions, journaux). Dans un second temps, certaines Églises évangéliques et organisations catholiques telles que la Jeunesse Estudiantine Catholique emploient les médias sociaux comme tribune pour diffuser leurs activités et mobiliser leurs membres. Enfin, certains groupes comme le renouveau charismatique et l'Union des groupes bibliques emploient de façon plus marquée les potentialités interactives des médias sociaux en comptant sur l'effet amplificateur, tout en conservant une stratégie de communication très structurée. 
La distribution inégale de la production médiatique numérique est évidente et illustre l'ampleur de la fracture numérique dans la société burkinabè. La presque totalité des sites, pages et plateformes étudiées sont basées à Ouagadougou, en plus de viser spécifiquement les groupes d'élites et les jeunes scolarisés. Ainsi, les églises rurales et toutes les activités en langues nationales, soit l'essentiel de la vie religieuse, se trouvent occultés de l'espace numérique. Ainsi, si l'implantation des Églises burkinabè dans la sphère numérique est un phénomène réel et en rapide évolution, il n'en reste pas moins qu'elle offre une vitrine sur un pan seulement de la vie religieuse d'une élite urbaine.

\section{Bibliographie}

BALIMA S., Frere M.-S. (2003), Médias et communications sociales au Burkina Faso. Approche socio-économique de la circulation de l'information, Paris, L'Harmattan.

BARZILAI-NAHON K. (2008), « Towards a Theory of Network Gatekeeping: A Framework for Exploring Information Control », Journal of the Association for Information Science and Technology, vol. 59, $\mathrm{n}^{\circ}$ 9, p. 1493-1512.

BRUNEL M. (1996), Les relations entre l'Église catholique burkinabè et le pouvoir de 1960 à 1995, Mémoire de DEA, Bordeaux, Sciences Po Bordeaux.

CAmpbell H. (2017), « Surveying Theoretical Approaches within Digital Religion Studies », New Media er Society, vol. 19, n 1, p. 15-24.

CORTEN A. (2006), « Un religieux immanent et transnational », Archives des sciences sociales des religions, $\mathrm{n}^{\circ} 133$, p. 135-151.

CSORDAS T. (2009) «Global Religion and the Reenchantement of the World: The Case of Catholic Charismatic Renewal », in T. CSORDAS (dir.), Transnational Transcendence. Essays on Religion and Globalization, Berkeley, University of California Press, p. 73-96.

Csordas T. (1994), The Sacred Self: A Cultural Phenomenology of Charismatic Healing, Berkeley, University of California Press.

FANCELLO S. (2006), Les aventuriers du pentecôtisme ghanéen. Nation, conversion et délivrance en Afrique de l'Ouest, Paris, IRD-Karthala.

FANCEllo S., Mary A. (dir.) (2010), Chrétiens africains en Europe, Prophétisme, pentecôtisme et politique des nations, Paris, Karthala.

GoRdon T. (2005), « Introduction: Visual Cultures of Pentecostalism», Material Religion: The Journal of Object, Art and Belief, vol. 1, n 3, p. 308-312. 
HACKett R. (1998), « Charismatic/Pentecostal Appropriations of Media Technologies in Nigeria and Ghana », Journal of Religion in Africa, vol. 28, n 3, p. 258-277.

KANE I. (2016), « Les catholiques sont l'élite ! Représentations de l'espace politique par une minorité religieuse au Burkina Faso », Canadian Journal of African Studies/Revue canadienne des études africaines, vol. 50, nº 1, p. 65-86.

KIRCHLEGER P.-Y. (2015), « At the Helm of the Number One French-Language Protestant Network, Jesus.net », Online - Heidelberg Journal of Religions on the Internet, vol. 8, p. 42-55.

LAURENT P.-J. (2009), Les pentecôtistes du Burkina Faso. Mariage, pouvoir et guérison, $\mathrm{Pa}$ ris, Karthala.

LEWIN K. (1947), « Frontiers in Group Dynamics II: Channels of Group Life; Social Planning and Action Research », Human Relations, vol. 1, n 2, p. 143-153.

MADORE F. (2016), La construction d'une sphère publique musulmane en Afrique de l'Ouest, Québec/Paris, Presses de l’Université Laval/Hermann.

Martin D. (2001), Pentecostalism: The World Their Parish, Oxford, Blackwell.

Meyer B. (2009), « Pentecostalism and Modern Audio-Visual Media », in K. NJoGU, J. Middleton (dir.), Media and Identity in Africa. Édimbourg, Edinburgh University Press, p. 114-123.

MOSSIERe G (2014), « Réseaux pentecôtistes, activités d'évangélisation et émotions partagées parmi les Congolais établis à Montréal : "un cosmopolitisme de charisme ?” ", in Y. FER, G. MALOGNE-FER (dir.), Le protestantisme évangélique à l'épreuve des cultures, Paris, L'Harmattan.

OosterbaAn M. (2017), Transmitting the Spirit: Religious Conversion, Media, and Urban Violence in Brasil, University Park, Penn State University Press.

SAMSON F. (2008), « Entre repli communautaire et fait missionnaire : deux mouvements religieux (chrétien et musulman) ouest-africains en perspective comparée », Social Sciences and Missions, vol. 21, n² 2, p. 228-252.

SAVADOGo M., Gomez-Perez M. (2011), « La médiatisation des prêches et ses enjeux : Regards croisés sur la situation à Abidjan et à Ouagadougou », ethnographiques.org, $\mathrm{n}^{\circ} 22$.

ShoemaKer P. J. (1991), Gatekeeping, Newbury Park, Sage. 
